\title{
Current State and Challenges in the Implementation of Smart Robotic Process Automation in Accounting and Auditing
}

\author{
Max Gotthardt, Dan Koivulaakso, Okyanus Paksoy, Cornelius Saramo, Minna Martikainen, Othmar \\ Lehner* \\ Hanken School of Economics, Helsinki
}

ARTICLE INFO

\section{Article history:}

Received 15 April 2019

Revised 11 June 2019 and 17

January 2020

Accepted 14 May 2020

Published 27 May 2020

\section{Keywords:}

Robotic Process Automation

Artificial Intelligence

Financial technologies

Accounting

Auditing

Technology implementation

Black Box Solutions

\section{ABSTRACT}

Technology development has grown rapidly in the last decades and gained importance for accounting and auditing through its identified potentials. Particularly the automation of judgment systems and systems that require human intervention, are deemed to be more relevant to confront a transformation through Robotic Process Automation (RPA). During the continuous development, the augmentation of such systems through Artificial Intelligence (AI) presents a greenfield project with high expectations. However theoretical frameworks have not yet been elaborative and sufficient to capture how such deployments can be conducted. Addressing this research gap, this study presents a summarized overview of the transforming RPA ecosystem and indicates what challenges are critical to being confronted for a successful implementation of such systems in accounting and auditing.

\section{Introduction}

In this article we discuss the use of RPA and AI in accounting and auditing. The intelligent automation of work has been a topic of discussion for over 20 years, however the implementation of RPA and AI systems in companies is still in its infancy. Only 15 percent of companies consider themselves to be mature in their use of RPA, and only 5 percent in AI (PWC 2017). Companies are still far from utilizing the vast opportunities provided by automation. As the capabilities of technology are improving in a rapidly increasing pace, it is vital for companies to adopt them successfully.

More specifically, we examine the challenges involved in the implementation of AI and RPA systems and the important factors that companies need to consider. Existing research has so far looked at the use of ERP systems in companies and its limitations (Hyvönen et al., 2008, Andersson et al., 2018)). Some research has also been done regarding the use of RPA particularly in the in the accounting profession and some of the challenges and opportunities involved (Cooper et al. 2018). However, a focused overview at how to successfully implement RPA and AI systems in accounting and auditing has been missing so far.

In order to fill this gap, we first introduce the relevant terminology needed to comprehend the possibilities of AI. We then give a brief overview of the current developments and research that has been done on the broader field. Next, we examine the challenges of AI implementation more in-depth. A typical framework of RPA is presented, and we

\footnotetext{
* Corresponding author. 
provide two case studies, one of a global software firm and one of a small Finnish payroll company to give a more practically oriented view of the present application of AI and the challenges these companies face.

We then attempt to identify the main challenges of AI implementation and indicate some suggestions for the practice. This will provide value to practitioners and academics likewise as we provide aggregated knowledge about the typical difficulties that companies are confronted with in their use of AI systems. This article can also serve as a checklist to practitioners when adopting new technologies in their company.

\section{Terminology}

Robotic Process Automation (RPA) and Artificial Intelligence (AI) are two closely knit terms that both have had and will continue to have a big impact on accounting and auditing practices. RPA and AI are on two opposite ends of an intelligent automation continuum. Automation is quickly moving from a process-driven to a more data-driven kind. Whereas RPA is highly process-driven, i.e. automating rule-based tasks, AI requires good-quality data for the AI to be able to learn what to do (CFB Bots, 2018).

The use of RPA has become widespread in the last 20 years and is a useful tool in all sorts of business administration tasks. RPA is a technology that automates standardized and rule-based activities using scripts. Robots can e.g. be used to copy and paste data between applications such as invoicing, payrolls or others (EY 2018d). Conventional RPA processes are constrained by technical capabilities and the judgment needed in operating them. Robots are not capable of handling unstructured data such as scanned documents etc. Humans are still needed to manually feed the robot with processed data which maintains low-value tasks for employees. There are also constraints in automating cognitive tasks. Several tasks exist that cannot be automated with current technology. These "cognitive" tasks have rules that can't be modeled because they require the experience of staff. An example is a request in an email, which requests to prioritize and making decisions (Perrier 2018).

RPA is sometimes misconceived as "old technology" that is being replaced by AI, which is a misperception. The two technologies do not replace each other but can be used separately or together. AI and RPA tools may increase the value of each other. For example, AI does not need RPA for creating insights from credit risk modeling, but RPA can be used to action the insights (EY 2018c).

Artificial intelligence, in fact, covers a number of interlinked technologies including data mining, machine learning, speech and image recognition, and semantic analysis. Data mining uses a combination of statistics, machine learning and AI to discover patterns in large data sets. This is important as the amount of data constantly increases, and unstructured data makes up $90 \%$ of all data. Data mining helps to find relevant information more quickly (SAS Institute 2019).

An important augmenting technology for $\mathrm{AI}$ is $\mathrm{OCR}$, which can be used to convert typed or handwritten text into machine-encoded text. This allows the firm to eliminate time-consuming manual data entry. Another central part of AI is Machine learning (ML). ML systems get trained by absorbing the learnings from the data and decisions and can make simple decisions and classifications. ML can, for example, be used in fraud detection. PayPal uses ML tools to separate transactions such as money laundering from legitimate transactions (EY 2018d).

Natural language processing (NLP) uses learning algorithms to analyze text and unstructured data. Natural language generation (NLG) is a technology that generates text or speech from structured information. It can be used to generate financial analysis reports, reporting numbers about a company's performance. Chatbots use both NLP and NLG to interpret voice or text and respond with standard predefined answers. Chatbots continuously learn new vocabulary to interpret the unstructured information. They are typically used in customer service functions to answer queries (EY 2018d).

\section{Implications in practice}

Currently, only 5 percent of companies consider themselves to be mature in their use of AI and 15 percent in their use of RPA (PWC 2017). This means that AI is still in its very early stages of adoption for broad society changing use. This partially depends on costs related to the adoption of such technologies and perhaps lack of skills within companies. However, the huge potential rewards of using AI and RPA technologies means that companies ought to gain expertise in the field.

AI and RPA together mean that a large amount of data can be turned into actionable insights, which allows full automation of entire processes. Investing in intelligent automation is very costly so it is of great importance to consider what combinations of RPA and AI are most suitable for the company (EY 2018c). This requires the 
knowledge of accounting professionals who are both experts in the business end but also in the technologies that are available.

PwC (2017) has estimated that $45 \%$ of work activities can be automated and that this will save $\$ 2$ trillion in global workforce costs annually. This shows how much companies can benefit from effectively implementing AI technology. It is strategically important for companies to start investing in innovation as AI is increasingly being adopted and the ones that have been successful in their implementation will make huge productivity gains in their work.

Automation through AI and RPA will also bring big changes to the accounting and auditing industry. Mundane and repetitive tasks typically done by junior staff will be taken over by robots. Instead, human expertise is needed at a higher level to make decisions that require judgment. RPA also brings great opportunities for internal auditing. Tasks such as testing of controls can be automated through RPA which expands the capacity of internal audit and frees auditors to more value-creating activities. Automation allows testing of full populations of data instead of sampling which increases the accuracy of auditing exponentially.

\section{Methodology}

For this analysis, relevant literature has been identified and filtered through the technologies and industries, that have been deemed relevant for the research question of presenting the current state and challenges in the implementation of RPA systems. Furthermore, an elaborative literature review has been pursued with a focus on analysis and summary of the key factors in practice rather than reflecting solely on the theoretical models. In order to capture the practical implications validly, two use cases have been carried out and an extended interview on the topic has been conducted, which has also been integrated into the argumentation of this paper. Finally, results have been reviewed by peers and a research framework has been created to present the findings.

\section{Current developments and ongoing research}

Organizations suffer from inefficient processes for accounting and auditing throughout different working units, which makes it harder to allocate scarce resources and targeted decision making. Currently used spreadsheets tend to be chaotic due to the heavy involvement of manual processes and introducing high potential for lags, errors and outdated data, which can be "referred to as 'shadow systems' as they are not formally recognized as part of the enterprise system" (Drum and Pulvermacher 2016, p. 181). Improperly created systems and insufficient quality and quantity of data with structural disproportions in databases and spreadsheets can cause significant financial losses such as the recent error that costed USD 100 Million for the shareholders (Tan 2014). Therefore, new technologies and frameworks are needed to solve these problems and fill in the gaps of sustainable management of resources and quality of processes.

Building on complementary technologies including Big Data and Blockchain, automation is predicted to have one of the highest impacts to the field of accounting in the short term. As discussed earlier, the emergence of this technology is inevitable considering the technology transformation in accounting. This transformation has been foreseen and analyzed for a long time. In the 1950s, the vice president of the National Cash Register Company, C.L. Keenoy, commented on this: "....We do know of course that we are in the early stages of an accounting and business management revolution which, in some respects, will rival the industrial revolution in its effect on the lives of everyone..." (C.L. Keenoy 1958, p.230). Originating from the automatic recording of punched cards and their processing in bookkeeping, the use of electronic technologies was recognized as revolutionary, while particularly the development of practice concepts for such an Integrated Data Processing (IDP) has been deemed critical. In alliance with this, the expansion of AI and other expert systems to automate accounting-related tasks can be traced back to the 1980 (Baldwin et al. 2006) and has been gaining further importance ever since.

Despite being identified as critical technologies decades ago, there is no clear consensus of how such deployment processes should be conceptualized in current practice and what the tangible challenges are there to be faced. In the following, we will elaborate more on how such a project structure can be summarized in the accounting and auditing fields.

A relevant question to ask before approaching RPA in auditing and accounting is whether or not to separate these two subparts from enterprise resource planning (ERP) or general corporate financial administration or see them as mere subparts to the big picture. When looking at financial administration more broadly (auditing, management accounting, book-keeping, personnel administration, salaries, tax payments etc.), it becomes evident that there are 
numerous interfaces that need to be compatible with each other for relevant data acquisition/collection to be possible. This requires legislative and operational change from official institutions as well as application in companies.

Hyvönen et al. (2008) indicate that ERP systems have brought a new type of concept to the digital transformation journey, integration. To exploit the large variety of functional autonomies in an organization, ERP systems have served as a bridge between these functionalities to enable a cross-functional and process-oriented approach (Quattrone and Hopper 2005). With this central control system, the CFOs had the chance to fulfil the role of being the "captain of the ship" and manage both the design and implementation models of the system (Andersson et al. 2018). However, Hyvönen et al. (2008) highlight the solution being prone to "complexity, which is evident from the numerous links to other information systems, updates of versions, data collection problems, the need for check-ups, arranging information storage, etc" (p. 46). It is also harder to reach high automation rates in accounting for particular cases such as accounts payable/receivable or auditing through ERP systems, which makes them obsolete for in-depth tasks requiring advanced judgment models.

Interviews with public accounting professionals from the Big 4 conducted by Cooper et al. (2018) reveal that there are often challenges related to the reluctance of the firms' clients to use RPA software. This is in part due to clients not completely understanding the capabilities of bots or how they operate. There is also some resistance because they are afraid that automation will negatively impact their employees' position. In addition, many clients are reluctant to adopt new technologies because of data issues. There are concerns related to the protection of business processes and the flow of information between different jurisdictions (Cooper et al. 2018).

\section{Framework}

Despite the narrative in the research on possible models based on RPA, a consensus hasn't been met in practice yet. To fill in this gap, the depiction of a typical back-office process in the background for invoicing of a purchase order by Deloitte (Schatsky et al. 2016) is guided analytically below. These operate first with human-led manual process, second with an RPA implementation and lastly with the augmentation of cognitive technologies for RPA.

In a manual process, the user periodically logs into the system and check orders, and once a request is confirmed, the purchase order is validated. Afterwards, specific pricing or discounts are applied to the order based on the client's contract. On top of this, additional changes are applied, that are outlined in the protocol and the purchase order is shipped and invoiced. This process is prone to human error and can bear judgmental complexities.

This process can be automated by an RPA robot, which is built on a software solution. This software first extracts data from the customer system and check for new orders. After downloading the purchase order, it is redirected into the legacy system. Here a human supervisor validates the order for its validity and accuracy according to the customer's contract terms. Afterwards, the process continues with the automated robot uploading the purchase order to the internal ERP system, for instance SAP or Oracle. Here discounts are adopted automatically based on customer agreements. The software would again pull data from customer system and check for orders, and once it is downloaded, they are pushed into the legacy system. Customer contract is processed with NLP to extract relevant information and match them. Afterwards, the discounts follow automatically based on customer agreements.

In the automated version of these three working concepts the human role is limited to supervision, and the system will be further prone to cost savings and efficiency gains due to the automated training and learning path, which is much harder for humans. Interestingly, Baldwin et al. (2006, p.79) indicate that auditors prefer human processing to decision aids or knowledge-based systems. This shows that a proof-of-concept (POC) in particular cases may support the company benefits of such systems and align on possible improvements through automation. Depending on the complexity and scope, Schatsky et al. (2016) indicate that a POC may take around two weeks, whereas a pilot can be operating within four to eight weeks.

\section{Case studies}

In order to present current applications in the market, we will present a macro view of one of the global leaders in shared services and RPA robot developers and a micro view of a startup ecosystem in Finland. Thus, in this macro view, we focus on the analysis of the technologies and the collaborative ecosystem based on automation, whereas the micro view captures the societal implications and the role of automation in a local setting for innovation. This distinction provides the differences in approaches in environment, implications and solution concepts for further research. Additionally, we briefly present their automation ecosystem and indicate what challenges can be relevant. 


\section{Case 1: How to shape a collaborative landscape for RPA and AI in accounting and auditing}

Founded in Romania in 2005, UiPath started out with software development kits (SDKs). Growing from 1 million USD to 100 million USD annual recurring revenue within 21 months, they have been counted as one of the fastest growing enterprise software companies (UiPath 9/18/2018). Having raised around 450 million USD funding up to date with their latest valuation at 3 billion USD through important venture capital funds, including Sequoia Capital, Accel, Earlybird, Kleiner Perkins and Seedcamp, they count 2100 global customers up to date and are acquiring 6 new customers per day. With a developer community of more than 250.000 peers worldwide, their platforms follow high usability marks and a direct market approach through large accounts across different industries.

The robot will open the invoice's folder in the mail and the PDF document will be opened in background to extract the relevant information. These include company name, invoice date, invoice reference number, description, taxable amount, tax rate, total amount and contact information. The UiPath runs the robot, which opens the email, saves the attached invoice and extracts the needed information, while highlighting the activities being executed. After reading the text from the PDF, information is extracted from the file. The robot also performs data conversion and format changes in order to validate the input in SAP. A screenshot of this process in UiPath Studio can be seen in Figure 1. After this extraction is done, the robot logs into SAP with encrypted login data. Then a search is conducted if there are entries for this vendor in the system, as depicted in Figure 2. In that case, certain information can be adapted from previous internal data. Afterwards, the invoices are entered with data extracted from the files. Finally, the VAT information is checked with the invoice and database. Cases can be defined in order to send the invoice to a predefined user and to ask what the robot should "post" or "park" it into the system. Then the original file is moved to processed invoices folder in the mail system. Through such an automation approach, clients can majorly eliminate human intervention in invoice processing.

This invoice processing concept with UiPath Studio has been deployed with a client in automotive manufacturing (UiPath 2018), where the client faced challenges to pursue frequent manual interventions for reading, validating, registering and posting of invoices. As the number of invoices to be processed per day was at 2.000, the decision was made to automate this process. Through the solution time savings of manual work was at $65 \%-75 \%$, suboptimizations were avoided as the automation is controlled from a central system and a structure was brought into the processing concept of the client.

See Annex 1 for screenshots of the workflow.

\section{UiPath Business and Technical Analysis}

There are several ecosystems, which have enabled the growth of UiPath, according to the Chief Evangelist Guy Kirkwood (Baxter 2018). First, their clients applying RPA to develop automated processes account for the highest transaction potentials. Second, they have technology partners including Google Cloud, IBM Watson, Celonis with their capabilities in complementary technologies including NLP, OCR, ML, which are integrated to the value offerings of UiPath Orchestrator, their central control system. It can be argued that their multitenancy and backward compatibility capabilities can be seen as a unique selling proposition (USP), which other players in the industry struggle to develop (Le Clair 2018). Third, software companies including Microsoft, Google and Oracle and service providers such as Salesforce are integrating the company's offerings into their platforms as to expand their reachability to further business operations. Such technical syndications can enable them to build on to their current offerings, for instance, developing a process mining product based on their cloud platform will give them an advantage against a competing automation provider. This can be augmented by their different cognitive solutions including ABBYY FlexiCapture to work with unstructured data. Fourth, system integrators including Deloitte, Capgemini, Accenture, KPMG utilize UiPath's solutions for their clients during their digitalization journeys for automation projects due to high returns in their return on investment (ROI) and lower costs.

UiPath should improve their automation reusability (e.g., for error handling and logging) versioned within the platform (Le Clair 2018, p. 12). Their success factors depend highly on the operation of their direct market model, system training and how they scale. Therefore, their partnerships in training including RPAbox and Symphony are critical for the success of system deployment, as this is one of the most significant challenges in the technology implementation. 


\section{Case 2: A micro view on how to utilize RPA and AI in auditing and accounting}

This chapter examines RPA in auditing in relation to broader business administration tools based on an interview with the payroll company Salaxy.com (Isosävi 2019) and has its main focus on the social implications identified by the company and how they approach technology innovation in financial industries. Salaxy offers a real-time salary payment solution with an automatic and intelligent payroll and has some of the biggest Finnish financial companies as its clients.

Currently, it may be argued that AI is overemphasized as a buzzword, whereas the majority of what is commonly referred to AI in normal process automation without machine learning. It needs to be remembered that, while some of the biggest proponents of the hype are the Big4 and their reports, they are also advertising, branding and selling their own tax and general consultation services.

One reason for this is the difficulty of acquiring relevantly structured data to learn from. A vast proportion of businesses have a lot to do in the basics of automation for AI to be relevant for them. Just combining accounts payable and due, taxes, invoices and salaries in one user interface would be revolutionary for small business (Isosävi 2019).

The national income registry that Finland has taken in use 1.1.2019 is a good example of only having one specific and not a broader societal mission of enabling automation. The registry only houses salary payment and tax information and not several other things related to paying someone's salary such as e.g. household tax-deductions. This is because it is built only from an income tax point of view. The registry is also an example of process automation being not yet suitable for AI solutions because it is not designed to be a modern data storage as it could have been. Without a holistic view on data and systematic collecting of it, not only present but a future application will be left undeveloped. This is due to the fact that the amount of possible AI solutions increasing together with more interfaces to combine existing structured data. A revolutionizing concept would be to combine the income registry with a realtime Value Added Tax (VAT) -registry to give the real-time "pulse" of the economy. Any arguments on how the economy is doing would be rendered useless with such a tool.

The big societal discussion should not be only about having a holistic view, but also about what kind of data is gathered and who administers it. In order for data security and privacy to be secured legislation and standards need to be put in place for how to administer data and metadata and for how to access this data while also securing everything is logged for oversight. States looking at user needs instead of foundations and principles easily leads to miscalculation such as in the case of the Finnish Transport and Communications Agency.

There are of course some AI in place already to justify some of the hype. Looking at bigger business and financial institutions some of the most advanced AI solutions and also examples of legislative activity in enabling use of AI can be found in banks and AML practices. How banks recognize patterns from money transactions to flag laundering and other illicit transactions are based on activities related to EU's 5th AntiMoney Laundering Directive. The automatic processes put in place in banks have also become tools for gathering data that is needed for the machine learning to further enhance AML-practices.

\section{Future Potential}

Robotics and intelligent automation house huge potential for financial accounting (EY 2018c). However, traditional RPA has limits, as it can only process information in digital form and not e.g. check things over the phone or through informal interaction. According to Ernst \& Young more digitalization and especially machine learning is needed for it to reach its full potential and value creation (EY 2018d).

Looking at digitalization in depth (E, (2018d) has described how to make clients change the way they work in order for them to deepen their digitalization capabilities. According to EY (the process of automation needs digital enablement. This means that in order to automate anything clients need to digitalize and decipher unstructured data gathered through e.g. digital forms, chatbots and voice recognition and convert it to structured data as a prerequisite for any form of robotics (RPA), which again is the prerequisite for AI (e.g. intelligent optical character recognition. Further to make the most of automated processes the digital workflow needs to include agent portals that may interact with people.

Robotic Process Automation (RPA) or digital labor or the use of software robots to automate processes are easy to configure and may deployed to automate manual tasks. They can perform activities such as copying and pasting data between applications, reconciling and cross-referencing data between different systems and conducting highlevel (PWC 2017). 
Within the internal audit, new testing approaches are needed for automated processes. This comes with an urgent need to also understand risks introduced by RPA and ensure that controls are well designed and effective to mitigate risks of new tech. Automation can increase compliance and reduce risk. Machines, unlike humans, do not skip a process step or become fatigued when going over transactions. Results are also always standard free of bias or variation which makes them accurate. On the other hand, this also means that possible errors become systematic and robots may not understand business environment changes that humans would notice. This means RPA governance needs to be put in place.

Beyond the automation of controls testing, RPA offers significant potential to change internal audit. Possible tasks include tracking and monitoring key risk indicators (KRIs), automated reporting and dashboard conception, shaping balanced scorecards and more (PWC 2017). In addition, the evaluation of data quality through master data and the assessment of administration through log files increase the validation of systems and gives control to the users.

Further, benefits of RPA may only be realized when deployment is managed with the same discipline and consideration as any other technology-based project. Internal audit needs to leverage companies' digital initiatives as a technology platform to reduce cost and increase risk coverage (PWC 2017).

Using advanced RPA methods enables the use of artificial intelligence through machine learning. When teaching machines any flaws in data will naturally severely flaw the functionality of algorithms and such further emphasizing the importance of RPA governance as the robotic processes are the sources of gathering data.

General trends of artificial intelligence are of special relevance to financial transactions as vulnerabilities in these would be a lucrative target for cybercrime. Further, anyone not wanting to give a true and fair view of their concern might want to rig AI used for auditing. This means documentation and logs for user interface and other software needs to be extremely transparent when any personal information is processed. A worst-case scenario of potential problems can be found e.g. in the vulnerabilities of the digital driver's license service launched by Finnish Transport and Communications Agency (YLE 2018). Besides transparent software solutions also relevant and cleanly scraped data are of essence for any AI solutions in finance.

When looking at rapidly changing opportunities through digital services it is also important to bear in mind that we are in a process of change and that the pace of change is still not clear. Looking e.g. at how digitalization has affected the Finnish financial giant Nordea they have invested 730 million euros in compliance and functional security as well as hired 1300 compliance experts and trained 12000 customer service professionals for 110000 hours in 2018. In the meanwhile, the capacity of the company's robots now adds up to the equivalent of 1500 personnel and it has invested over 200 million euro in digital services (Kauppalehti 2019). This shows that full automation is far from here.

\section{Discussion and analysis}

As the area of research is still relatively new, the amount of identifiable challenges and risk factors are many and of various consequence. In the following chapter we present a few of those factors that we have deemed to be critical in both the existing RPA systems and implementation of new ideas. These challenges have been identified by the Big 4 auditing firms and smaller solution providers and integrators. An overview has been created by the study based on the academical literature in line with the industrial argumentations from Deloitte (2018) and PWC (2017) in order to reflect different aspects of risks of implementing an RPA programs augmented with AI systems (see Annex 2). These have been captured mainly from the business and technical approaches and should be further augmented with societal views presented above. Therefore, originating from these findings on challenges, significant focus fields are presented for a discussion for future research and industrial investigations. 
Table 1. Identified targeted risk categories for implementing a program with RPA and AI (Edited, augmented and modified from PWC (2017) and Deloitte (2018))

\begin{tabular}{|c|c|c|c|}
\hline \multicolumn{2}{|r|}{ Business Risks } & \multicolumn{2}{|c|}{ Automation Risks } \\
\hline $\begin{array}{c}\text { Change } \\
\text { Management }\end{array}$ & $\begin{array}{l}\text { How is HR tackling the impacts of RPA? } \\
\text { How are changes to be communicated? }\end{array}$ & $\begin{array}{l}\text { Identity and access management } \\
\text { Secured business processes }\end{array}$ & $\begin{array}{l}\text { Center of } \\
\text { Excellence }\end{array}$ \\
\hline Executive & $\begin{array}{c}\text { Who has the ownership of RPA initiatives? } \\
\text { Who will manage the framework and } \\
\text { promote efficiencies? }\end{array}$ & $\begin{array}{l}\text { License Compliance } \\
\text { Automation strategy and governance }\end{array}$ & $\begin{array}{l}\text { Proof of } \\
\text { Concept }\end{array}$ \\
\hline Functional & $\begin{array}{l}\text { Who designs control systems? } \\
\text { Are there scalability limitations in RPA and } \\
\text { core systems? }\end{array}$ & $\begin{array}{l}\text { Adaptation schemes of existing systems } \\
\text { with new features } \\
\text { Legacy systems for simultaneous and } \\
\text { unified operations across technical } \\
\text { testing and rollout }\end{array}$ & $\begin{array}{c}\text { Backward } \\
\text { Compatibility }\end{array}$ \\
\hline Technical & $\begin{array}{l}\text { How will the data quality and accuracy be } \\
\text { ensured? } \\
\text { How is the tests, validations, maintenences? }\end{array}$ & $\begin{array}{c}\text { Incident management and business } \\
\text { continuity } \\
\text { Regulatory compliance }\end{array}$ & Implementation \\
\hline Operational & $\begin{array}{l}\text { What controls exist to monitor } \\
\text { performance? } \\
\text { How will the business comply with } \\
\text { regulatory requirements? }\end{array}$ & $\begin{array}{l}\text { Data leakage and privacy } \\
\text { Cyber threats }\end{array}$ & $\begin{array}{l}\text { Business } \\
\text { Case }\end{array}$ \\
\hline
\end{tabular}

\section{Only proper guidance leads to good results}

There is a major risk that occurs when an overhaul of a previous routine is conducted in the form of the approving manager not fully grasping the concept. The programmer or IT tech that builds or supplies the system that is meant to revamp the system in place has a perfect understanding of the ins and outs of the interface and underlying processes. This is most likely not the case of most upper-level managers as seniority in a company is often linked to an older generation which means a need for an in-depth education of general concepts that they have not dealt with in the past. As the level of knowledge is at a low point, the risk of misuse can be assumed to be high and faults occurring not due to the failure of the actual system but instead from upper management. These issues might not have occurred if an RPA system was not in use and for instance, the second tier of human interaction prevents this.

Having proper motivation behind the project is key. As the introduction of an RPA serves as a business project, the main focus has to be on the benefits from a business perspective. If the IT focus becomes too high, there is a risk that the end goal of the system is forgotten. This can be a factor in figuring out how the RPA is implemented into the existing workforce and to which degree it replaces conventional jobs.

An issue regarding AI systems is how easily they can be manipulated or broken to take on unwanted characteristics. As AI tries to emulate a humanlike thought process the risk of ruining it through stimulus is a factor in the planned use. "Tay" the Microsoft experiment in 2016 is a prime example of this manipulation. It took twitter under 24 hours to fully corrupt this AI bot that was emulating an average female user into spewing out sexist, racist and other unsavory comments in response to a dialog. This exemplifies perfectly one of the weaknesses of AI, data. If the quality of the data that is being used to learn from is weak most of today's AI systems are not able to get past this initial hurdle and recover (Sakata 2018).

\section{Cybersecurity and vulnerability through malicious AI systems}

Any attempt at cracking or hacking financial systems that can be automated with use the of AI learning systems are theoretically much more effective and harder to prevent. This is relevant in the financial industry as hackers can 
infiltrate key systems and remain there using AI learned behavior. EY identifies four different cyber risks that are tied to the implementation and usage of RPA:

Abuse of privileged access, Disclosure of sensitive data, Security vulnerabilities and Denial of service. These risks are factors that can be exploited by malicious users to access sensitive data that is being processed in an RPA system. But RPA systems are not different from other IT systems and countermeasures are readily available and under continuous development. There are other measures to take to ensure a safe system outside actual IT solutions, such as having strict control over who has access to the system and a transparent and traceable entity that can be monitored easily (EY 2018b).

\section{Correct usage and right strategies are critical}

As the financial sector is keen to be on the current trends and adopt methods that have shown to be successful in other companies and situations the risk of neglecting the true need of your company is high. This could lead to an almost impulsive choice of RPA in a situation where another system or even personnel solution is more cost-effective and/or easier to implement and use. Avoiding the trendy pick is often hard to do as it requires to distance yourself as a manager and seeing the bigger picture (EY 2018a).

\section{Future outlook}

\section{Implementation needs resource allocation}

Ensuring that an RPA is running at maximum potential is key in the implementation process of these systems. Enabling the RPA on an enterprise level for scheduling purposes and allowing it to do the tasks "unsupervised" are keys mentioned by the symphony ventures blog. These abilities are meant to save both time and money which is the bottom line of why RPA systems are being developed in the first place (Brain 2017). The running costs for the implementation of RPA systems in terms of timeline and costs are relatively insignificant when compared to major updates in conventional IT platforms in auditing systems.

There is a fear that RPA will be struggling with the often-occurring non-standardised invoices and data that it would need to handle. OCR systems and a hierarchical system of approval and exception that is flexible enough is required to cope with the demand of the accounting industry (EY 2016a).

\section{Financing and proof of concept (POC) needed}

There is much need for POC before any major decision is made in most businesses and RPA and AI is no different. Unfortunately, this is an issue when it comes down to breaking down cost-benefit calculations before implementation as true measurements can usually only be attained after the system has been observed in action. Developing an effective way to prove the usability of a system before major monetary commitment can be crucial for the implementation and mass-usage of a system. Especially beneficial POCs are those that are tested in a similar environment as the intended real-life system (Lang T. 2019).

\section{Black Box Solutions: Transparency needed}

To properly monitor the impact that an RPA has on the business the management must have the ability to judge it fairly, meaning that transparency is key. The goal of any RPA is to deliver on its intended task better than previous methods and ensuring that this is indeed happening is vital. (EY 2016b). Explainable AI is a field that intends to make AI decision-making more like human behavior patterns and thus creating a path that can be followed by an overseer. If the company understands the reasoning behind the AI, the transparency and usability of the system increases (Rao A. \& Golbin I. 2018). For this explainable and interpretable systems for the testing and deployment of AI can play an important role both to detect biases in the data, flaws in the model and gaining new insights to the system (Samek et al. 2017). 


\section{Adding solutions for the smaller players}

RPA service providers have often large concepts and projects in mind when pitching their ideas, but a large part of the accounting industry is still operating on a smaller scale. This opens a potential for the use of RPA or AI systems in start-ups and other smaller businesses where the need for accounting can be a problem cost-wise (CiGen RPA, Medium.com). Offering a clear path of processing financial tasks and allowing for internal growth can be a difference maker in setting up a new company.

\section{Limitations and further research}

This study contributes to academic research by implying relevant research fields in order to explain the current state of RPA and AI methodologies across the accounting and auditing professions. Besides elaborating on the challenges, the provided status quo on the technological applications have been presented with two cases to cover different aspects in practice. On top of the multi-factor qualitative analysis, this study delivers an overview on current state and categorized challenges of RPA and AI programs in a focused manner (see Annex 2), which should be further extended based on implementation fields and departmental structures. A major limitation is the scope of the study and the focus on the practical aspects rather than the theoretical implications. Originating from this, future research should combine novel academic publications with practical models. Furthermore, empirical studies on time savings and amount of human intervention should be conducted to identify areas regarding task complexity and intensity for manual work in order to indicate the fields where RPA provides the highest benefits. This should also include the testing based on cases to understand in how far the augmentation of RPA through AI systems provide additional benefits. Thus, this will help to identify business, technical and societal challenges in the implementation of such systems in both the micro and macro views. This study should be regarded as a starting point to assess the relevance of fields for further research to connect research and implementation in the industry regarding these technological models.

\section{Conclusion}

With our presented cases from the industry and reviews from research, we identify that the potential of RPA and AI in the field of accounting and auditing is increasing and is already being seized. Bresnahan et al. $(1999$, p.11) indicate that complex and cognitively demanding work or tasks that require judgment, creativity and frequent exceptions are remarkably hard to automate with computers (see Wilson and Sangster 1992), whereas important benefits are possible to seize with RPA systems augmented by AI. Baldwin et al. $(2006,82)$ suggest that AI researchers play a significant role to solve key issues about audit and assurance with the use of techniques including "fuzzy logic, neural networks and perhaps other areas of AI that have never before been applied in an accounting context". In current applications, we have presented that these applications already play an important role in the improvement of RPA systems, while also increasing the factors to consider. By automating a good deal of the manual work that requires a lot of personnel to complete through human intervention, the threshold to improve efficiency is lowered. While this presents a significant potential of time and capital savings, we have identified numerous challenges to be confronted, which are in line with the implementation experiences in the industry (PWC 2017). While AI systems will help answering important questions about data, the quality and quantity of data input for such systems is a critical question. Furthermore, opening the black box of AI is vital in order to deploy such systems with responsibility schemes and expect higher automation degrees. To fill in this gap, explainable and interpretable AI systems present a greenfield for transparent technology development. While the cyber-attacks are becoming more powerful and fraudulent activities are showing frequent progress, also cyber-defense is becoming stronger to support the system's security and safety. Government and intra-governmental regulation and registries have a huge impact on employers needing to renew their administration. It is important to understand that these improvements in automation require broad distribution of responsibility schemes, C.L. Keenoy (1958, p. 236) suggested many decades ago that these responsibilities will bring more opportunities in the forthcoming era of automation.

Throughout our research in the field of RPA and AI in accounting and auditing, we have presented technical, societal and business contexts on the state and challenges of current technology and implementation, where the main persistent issue in all cases has been the importance of the mindset and ecosystem modeling (see Annex 2). To achieve a successful implementation of such systems in accounting and auditing, it must be kept in mind that crossdepartmental support and clear mindset must be retained, both clear strategies and problem statements have to be 
defined and know-how has to be canalized into the right centers of excellence with right skills. This will help them mitigate the risks and ensure leaner processes when dealing with errors or insufficiencies. Only the organizations, which are considering the extensive amount of risk factors from different views, can exploit the full power of RPA and AI systems, whereas others will be investing scarce resources into untargeted and unreliable processes without significant ownership of business or technology segments.

\section{References}

Andersson, Per; Movin, Staffan; Mähring, Magnus; Teigland, Robin; Wennberg, Karl; McGettigan, Karyn (Eds.) (2018): Managing digital transformation. 1. ed. Stockholm: SSE Institute for Research, Stockholm School of Economics ([SIR:s årsbok, 2017])

Baldwin, Amelia A.; Brown, Carol E.; Trinkle, Brad S. (2006): Opportunities for artificial intelligence development in the accounting domain. The case for auditing. In Intell. Syst. Acc. Fin. Mgmt. 14(3), 77-86. DOI: 10.1002/isaf.277.

Baxter, Michael (2018): RPA market with UiPath in the cockpit is taking off like a rocket: Information Age. Retrieved from https://www.information-age.com/rpa-market-uipath-123476832/, checked on 2/2/2019.

Brain, David (2017). RPA Technical Insights, Part 19: Doing More for Less: How to Optimize Resource Allocation in RPA. Symphony ventures blog. Retrieved from: http://blog.symphonyhq.com/rpa-technical-insights-part-19-optimize-resourceallocation

Bresnahan, Timothy; Brynjolfsson, Erik; Hitt, Lorin (1999): Information Technology, Workplace Organization and the Demand for Skilled Labor. Firm-Level Evidence. Cambridge, MA: National Bureau of Economic Research.

CFB Bots (2018). The Difference between Robotic Process Automation and Artificial Intelligence. Retrieved from https://www.cfb-bots.com/single-post/2018/04/09/The-Difference-between-Robotic-Process-Automation-and-ArtificialIntelligence.

CiGen RPA, Medium.com "RPA for small businesses: 6 Gains it is likely to bring about" Retrieved from https://medium.com/@ cigen_rpa/rpa-for-small-businesses-6-gains-it-is-likely-to-bring-about-f50d62530677

C.L. Keenoy (1958): The Impact of Automation on the Field of Accounting: The Accounting Review (No.2), 1958, pp. 230236. Retrieved from: https://www.jstor.org/stable/241233?origin=JSTOR-pdf\&seq=1\#page_scan_tab_contents, checked on 2/6/2019.

Cooper, Lauren; Holderness, D. Kip; Sorensen, Trevor; Wood, David (2018): Robotic Process Automation in Public Accounting. Retrieved from: https://papers.ssrn.com/sol3/papers.cfm?abstract_id=3193222

Deloitte (2018): Auditing the RPA environment. Our approach towards addressing risks in a BOT environment. Deepa Seshadri; Ashish Sharma. Available online at https://www2.deloitte.com/content/dam/Deloitte/in/Documents/risk/in-ra-auditing-therpa-environment-noexp.pdf.

Drum, Dawna M.; Pulvermacher, Andrew (2016): Accounting Automation and Insight at the Speed of Thought. In Journal of Emerging Technologies in Accounting 13(1), 181-186. DOI: 10.2308/jeta-51441.

Hyvönen, Timo; Järvinen, Janne; Pellinen, Jukka (2008): A virtual integration-The management control system in a multinational enterprise. In Management Accounting Research 19(1), 45-61. DOI: 10.1016/j.mar.2007.08.001.

EY (2016a) Robotic process automation in the Finance function of the future. Retrieved from: https://www.ey.com/Publication/vwLUAssets/EY_Robotic_process_automation_in_the_Finance_function_of_the_future/\$ FILE/EY-robotic-process-automation-in-the-finance-function-of-the-future-2016.pdf

EY (2016b) Get ready for robots. Retrieved https://www.ey.com/Publication/vwLUAssets/Get_ready_for_robots/\$FILE/ey-get-ready-for-robots.pdf

EY (2018a) Risk and control considerations within robotic process automation implementations. Retrieved from: https://www.ey.com/Publication/vwLUAssets/EY-risk-and-control-considerations-within-RPA-implementations/\$File/EYrisk-and-control-considerations-within-RPA-implementations.pdf

EY (2018b) How do you protect the robots from cyber attack? Retrieved from:

https://www.ey.com/Publication/vwLUAssets/ey-how-do-you-protect-robots-from-cyber-attack/\$FILE/ey-how-do-you-protectrobots-from-cyber-attack.pdf

EY (2018c). Robotics and intelligent automation Combining the power of human and machine. Retrieved from

https://www.ey.com/Publication/vwLUAssets/EY-robotics-and-intelligent-automation/\$FILE/EY-robotics-and-intelligentautomation.pdf

EY (2018d). Intelligent automation Reshaping the future of work with robots. Retrieved from https://www.ey.com/Publication/vwLUAssets/EY_intelligent_automation/\$FILE/EY-intelligent-automation.pdf

Isosävi, Janne 2019. This chapter is based on a background interview on the topic done with co-founder and CMO Janne Isosävi of Salaxy.com on 8.2.2019 9.30-10.00. Salaxy offers Real-time salary payment solutions with automatic and intelligent payroll.

Juvonen, Anna Kauppalehti 6.2.2019 "Nordean von Koskull listaa muutosohjelman hedelmät: Robottien kapasiteetti vastaa 1500 henkilötyövuotta" (Kauppalehti 6.2.2019) Retrieved from: https://www.kauppalehti.fi/uutiset/nordean-von-koskull-listaamuutosohjelman-hedelmat-robottien-kapasiteetti-vastaa-1500-henkilotyovuotta/7d335225-3b76-471d-9b81-38fb0eef0e71 
Lang T. 2019, Enterprise services outlook "Executing a proof of concept test for robotic process automation" Retrieved from: https://www.esoutlook.com/cxoinsights/executing-a-proof-of-concept-test-for-robotic-process-automation-nwid-1161.html

Le Clair, Craig (2018): The Forrester Wave ${ }^{\mathrm{TM}}$ : Robotic Process. The 15 Providers That Matter Most And How They Stack Up, 2018, 1-23.

Perrier, F. (2018). Pushing the limits of RPA with AI. Retrieved from: https://www.capgemini.com/2018/11/pushing-the-limitsof-rpa-with-ai/.

PWC (2017) Robotic process automation: A primer for internal audit professionals. Retrieved from https://www.pwc.com/us/en/risk-assurance/publications/assets/pwc-robotics-process-automation-a-primer-for-internal-auditprofessionals-october-2017.pdf

Quattrone, Paolo; Hopper, Trevor (2005): A 'time-space odyssey'. Management control systems in two multinational organisations. In Accounting, Organizations and Society 30(7-8), 735-764. DOI: 10.1016/j.aos.2003.10.006.

Rao A. \& Golbin I. 2018 PWC: "What it means to open AI's black box" Retrieved from: http://usblogs.pwc.com/emergingtechnology/to-open-ai-black-box/

Sakata, Toby (2018) The Good, The Bad and The Ugly of Artificial Intelligence and Machine Learning. Medium.com. Retrieved from: https://medium.com/applied-innovation-exchange/the-good-the-bad-and-the-ugly-of-artificial-intelligence-andmachine-learning-3f7e663c317a

Samek, Wojciech; Wiegand, Thomas; Müller, Klaus-Robert (2017): Explainable Artificial Intelligence. Understanding, Visualizing and Interpreting Deep Learning Models, 8/28/2017. Retrieved from https://arxiv.org/pdf/1708.08296.

SAS Institute Inc (2019). Data mining. Retrieved from https://www.sas.com/en_us/insights/analytics/data-mining.html

Schatsky, David; Muraskin, Craig; Iyengar, Kaushik (2016): Robotic process automation. A path to the cognitive enterprise: Deloitte University Press (SIGNALS for Strategists), 2016, 1-10, checked on 27.01.19.

Tan, Gillian (2014): Spreadsheet Mistake Costs Tibco Shareholders \$100M: The Wall Street Journal, 2014. Retrieved from https://blogs.wsj.com/moneybeat/2014/10/16/spreadsheet-mistake-costs-tibco-shareholders-100-million/, checked on $3 / 1 / 2019$.

UiPath (2018): Case Studies. RPA at work. Focus on accounts payable: UiPath, pp. 1-2, checked on 1/24/2019.

UiPath (2018): UiPath Raises \$225 Million Series C Led by CapitalG and Sequoia. Irina Hutu. Retrieved from https://www.uipath.com/news/uipath-raises-225-million-series-c-led-by-capitalg-and-sequoia.

Wilson, R. A.; Sangster, A. (1992): The automation of accounting practice. In J Inf Technol 7(2), 65-75. DOI: 10.1057/jit.1992.11.

YLE (11.12.2018). Tietoturva-asiantuntija STT:lle: Trafin palvelun ongelmallisin seikka oli pelkällä henkilötunnuksella toimiva haku. Yle uutiset. Retrieved from: https://yle.fi/uutiset/3-10549455 
ANNEX 1

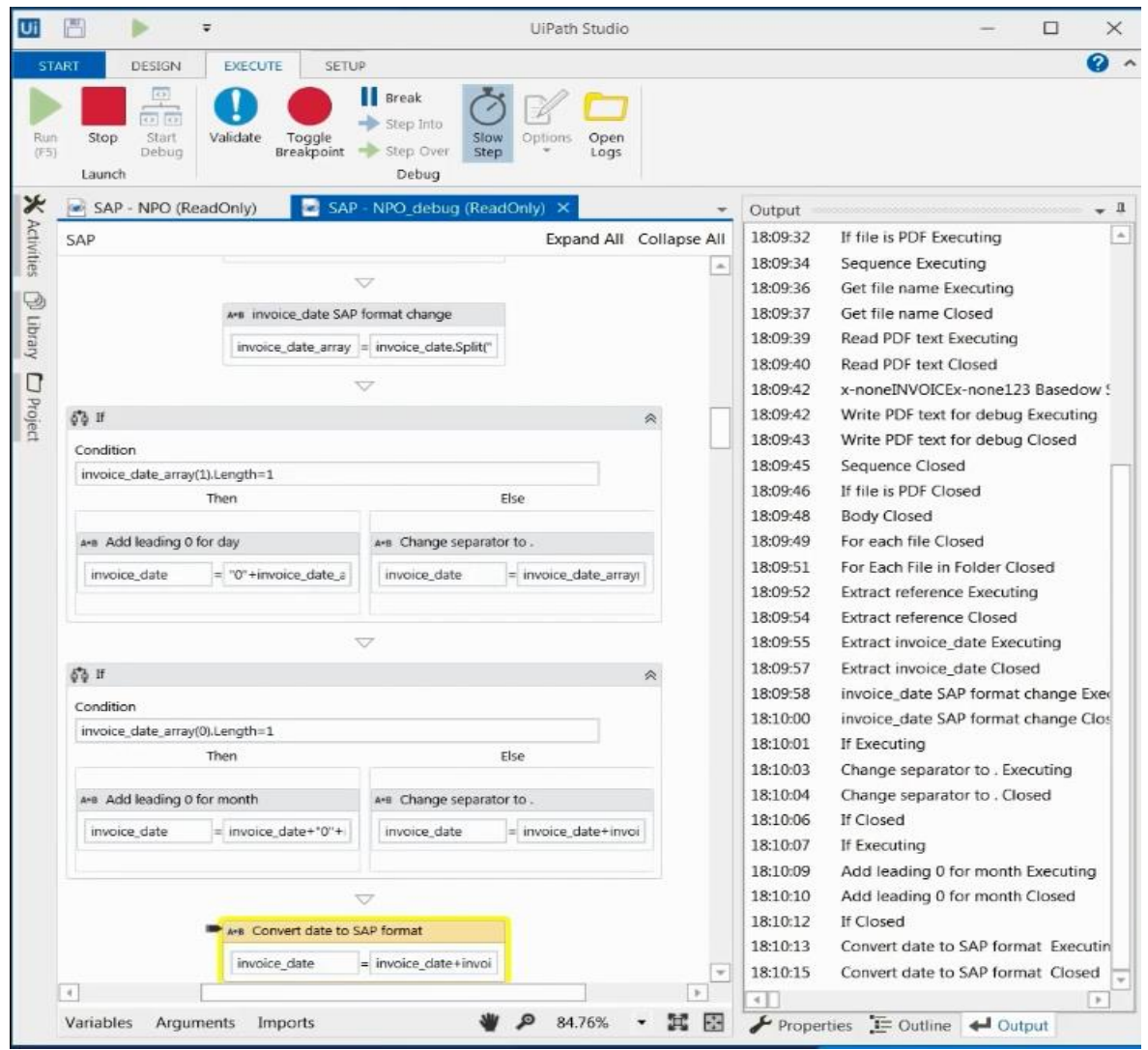

Figure 1. Screenshot of UiPath Studio for data extraction and conversion

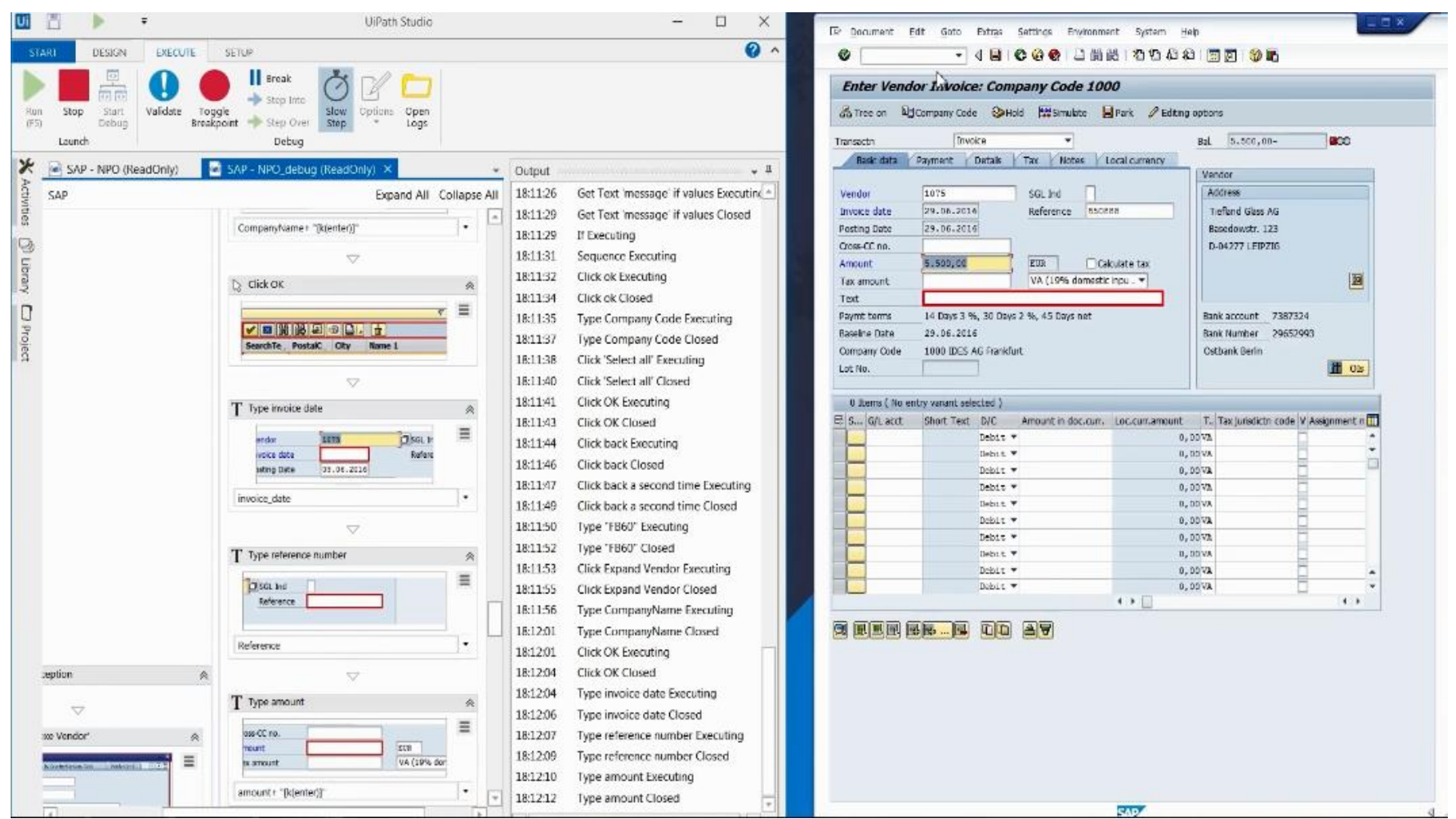

Figure 2. Screenshot of UiPath Studio for data entry and invoice processing in SAP 